\title{
How map features cue associated verbal content
}

\author{
SARAH E. PETERSON \\ Northern Illinois University, De Kalb, Illinois \\ and
}

\author{
RAYMOND W. KULHAVY, WILLIAM A. STOCK, and DORIS R. PRIDEMORE \\ Arizona State University, Tempe, Arizona
}

\begin{abstract}
Undergraduates learned a complex map, heard a related text passage, and received either the original map or a picture of its boundary as a retrieval cue for the text on both an immediate and a 2-week-delayed test. Features on the map were either modified by visually keyed adjectives or not modified. Recall of text events was higher for the map, for the modified features, and on the immediate test. Analysis of feature-event conditional probabilities indicated that a combination of feature icons and verbal modifiers provided the greatest recall of both modified and nonmodified events.
\end{abstract}

When a visual display works as a mnemonic for discourse recall, it generally does so by improving memory for information related directly to the elements of the display itself (Dean \& Kulhavy, 1981). This result has been replicated numerous times with geographic maps and related texts, but it also appears to hold for graphs and diagrams of various kinds. Improved recall of a related text seems to occur only when features are coherently distributed over the spatial domain of a map, and recall is best when the features themselves are represented as mimetic icons (Abel \& Kulhavy, 1986; Shank, 1990).

We have adopted the conjoint retention hypothesis as a way of explaining the improvement in text recall that results from the presence of a complex visual display (Kulhavy, Lee, \& Caterino, 1985). Conjoint retention involves both representational and computational assumptions, and it explains map-text facilitation in terms of links between image and verbal codes, as well as the efficiency with which attention can be moved across the map representation. In agreement with Paivio (1986), we assume that maps and text representations are stored in separate memory codes that are interconnected, so that information in one code can be used to activate information in the other. In addition, we believe that, with considerable effort, complex displays such as maps can be stored as relatively intact units so that they become synchronously available during retrieval. Hence, we assume that complex visual displays can be represented as relatively intact units in memory without loss of information about their component elements. Because the display is economically represented in active memory, the computational cost of search for individual features is reduced, and the change of attention from one location to another is com-

Correspondence should be addressed to Raymond W. Kulhavy, ISRF-0611, Arizona State University, Tempe, AZ 85287-0611. putationally easy (Larkin \& Simon, 1987). Under these conditions, the probability of being able to locate and use a visual feature increases as a function of the "intactness" of the representation. The accessed map feature then activates the feature label in the verbal code, and to the degree that activation spreads from the feature node to related verbal events, successful conjoint retention occurs.

In the present study, we furnished subjects at event retrieval with either a perceptual copy of a target map or a boundary of the initial map with the features missing. Of course, we expect perceptual maps to improve event memory relative to the memorial representation of the map, since all of the target features are immediately available. However, a comparison of the perceptual and memorial representations in terms of event recall allows us to evaluate the relative strength of the remembered image of the map.

We are also interested in the influence of visual detail, especially in terms of whether it is carried in the visual or verbal stimuli. To approach this question, we presented two events for each feature. In one event, the feature was modified with adjectives of size or shape, material, and color, whereas no such modification occurred in the second event. Elaborating a feature verbally in terms of its visual detail should increase the activation strength of the node representing the feature label. We expect this increase in activation strength to act additively with the icons in the perceptual map, increasing the recall of event information. Also, the strength increase should produce higher recall for nonelaborated events, simply because it yields a wider spread of activation from the label.

In addition to the main variables, we asked each subject to rate both the image strength and the locational accuracy for each feature on the map. The predictive value of such estimates is established, and we hoped to use them in teasing out the relative contributions of spatial and visual factors to conjoint retention. 


\section{METHOD}

\section{Design and Subjects}

Two forms of retrieval cue were varied between subjects, and both feature elaboration and test occasion were treated as within-subjects variables. The base design was a 2 (map cue: intact vs. boundary) $\times 2$ (feature elaboration: present vs. absent) $\times 2$ (occasion: immediate vs. 14 days) factorial, with repeated measures on the feature and occasion variables.

The subjects were $\mathbf{4 0}$ undergraduate volunteers attending a large public university in the Midwest, with about an equal number of males and females in the sample. The subjects were randomly assigned, 20 to each between-subjects condition, in the order in which they appeared for the experiment. Six subjects failed to appear for the second testing, so their data were eliminated from the analyses. The final numbers were 18 and 16 in the boundary and intact map conditions, respectively.

\section{Materials}

The intact map depicted a fictitious town named Fiori, which contained a total of $\mathbf{1 5}$ landmarks, as well as paths surrounded by a wall. Each map feature was identified by a mimetic line drawing and a label. The features represented entities commonly found in Italian hill towns (e.g., a fountain, plaza, temple, and market). The intact cue group saw the same map again at retrieval, whereas subjects in the boundary condition saw only the wall with all other feature information removed. The intact version of the map filled the $21.60 \times 27.90 \mathrm{~cm}$ sheet of white paper on which it was printed for use during the learning phase.

The text, written by the authors, consisted of a coherent 800 -word exposition covering both current and historical events occurring in the town of Fiori. With the exception of the "Wall," the features within the town were each mentioned exactly twice in the passage, with a different event linked to the feature in the same sentence each time it appeared. Each feature was presented once with, and once without, elaboration. Elaboration consisted of modifying the feature label with a word for a physical shape or size, as well as a construction material and a color. None of the descriptors were used for more than one feature. Examples of the elaborated and nonelaborated feature-event sentences for the feature "Temple" are shown below.

1. A portion of the temple was set aside for the worship of the ancestors of Fiori's population.

[nonelaborated version]

2 . The purple masonry of the narrow temple marked the spot where lightning started a fire long ago.

[elaborated version]

Feature-event sentences were randomly assigned to passage positions, with the restriction that no two occurrences of a given label appear sequentially, and that elaborated and nonelaborated sentences appear in alternate order throughout the passage. Filler sentences unrelated to the map occupied both the beginning and the end of the text. The text was tape-recorded in a female voice at a rate of approximately 100 words per minute for use in the experiment.

For the free recall test, the retrieval maps were photoreduced by $50 \%$, so that they could occupy the right-hand side of a $21.60 \times 27.90 \mathrm{~cm}$ sheet of white paper. Four such sheets were stapled together with their map to form a recall booklet. The subjects in the intact condition received the map seen during learning, but the subjects in the boundary condition saw only the wall printed on their recall sheet. Each recall booklet contained a front page of instructions, in which subjects were asked to recall, as accurately as possible, everything they could remember about Fiori.

Each subject completed two rating scales prior to the free recall test. The first scale, which was labeled "Visual Characteristics," contained the names of the 15 map features. The subjects were required to rate each feature name in terms of "how well they could see the feature in their mind." The ratings were produced using a 4-point, Likert-type scale, with 1 for "no image," 2 for "fair image," 3 for "good image," and 4 for "vivid image." The scale and instructions were both printed at the top of the page that contained the scale. The second scale was titled "Spatial Characteristics"; here subjects were asked to rate how accurately they could mentally picture where the features were located on the map. The scale points were 1 for "within four inches," 2 for "within two inches," 3 for "within one inch," and 4 for "within one-half inch." Again, both the scale and instructions were printed at the top of the page.

\section{Procedure}

The subjects completed the experiment in a university classroom, in which they sat as far apart as possible to reduce peeking. The subjects participated in groups of $10-15$, with about the same number of subjects from each map cue condition present at each session. As each person entered the room, he or she received an envelope from a randomly shuffled stack, which contained an assignment to a between-subjects treatment. The group was next informed about the nature and sequence of the study, and any general procedural questions were answered. Following this introductory period, the subjects removed their copies of the intact map from the envelope and were instructed to study the map carefully for $2 \mathrm{~min}$, and to pay close attention to the features and their arrangement. The subjects were also told that they would hear, and be tested on, a story related to the map, and that knowing the map would help them remember the events in the story. When the 2 -min study period was completed, a monitor instructed the group to continue viewing the map while listening to the recorded version of the "Town of Fiori." The recording was played twice, with the map-viewing instructions repeated between playings.

After the tape had been played, the group members completed example items from the two rating scales; then they removed the actual scales from their envelopes and were given unlimited time to finish the rating task. The order in which the scales were completed was counterbalanced across the two conditions. When the rating was completed, all scales were collected and replaced with two pages of complex arithmetic problems on which the subjects worked for $10 \mathrm{~min}$ in order to preclude recall from working memory.

Once the subjects had completed the interpolated task, they placed the sheets under their seats, removed either the intact or the boundary version of the test from their envelopes, read the front sheet of instructions, and were allowed to ask questions concerning general procedures. The subjects were given unlimited time to complete their free recall of the Fiori passage, with virtually everyone finishing the task within $20 \mathrm{~min}$. Each group was instructed to report to the testing site again in 2 weeks, but no specific information was provided concerning the delay task.

When the subjects assembled 2 weeks after the initial testing, they again received envelopes with their names; these contained both the rating scales and the test booklets appropriate to their respective conditions. The order of the two scales was the reverse of the initial rating for each subject, and the scales were completed in the same way as on the 1st day. As before, the subjects wrote down all that they remembered about the Fiori tape in booklets containing either the intact or the boundary maps. The free recall was not timed.

\section{RESULTS}

The free recalls were scored according to lenient criteria. Events were counted as correct if their substance was represented, and modifiers as correct if any one of the adjectives was recalled. One point was given for each correct response. All protocols were scored by one of the authors. A sample of 10 protocols was scored independently by a second evaluator; there was $94 \%$ agreement between the two sets of scores.

All statistical tests were evaluated at the conventional $p<.05$ level of confidence. A 2 (map) $\times 2$ (elaboration) $\times 2$ (occasion) unweighted means analysis of variance on the event recall data yielded significance for the main effects of map $\left[F(1,32)=4.57, M S_{\mathrm{e}}=22.01\right]$, elaboration $\left[F(1,32)=5.50, M S_{\mathrm{e}}=4.08\right]$, and occasion $\left[F(1,32)=127.09, M S_{\mathrm{e}}=1.75\right]$. There were no significant interactions. Inspection of the means and standard deviations in Table 1 shows that all main effects were in 
Table 1

Means and Standard Deviations for Correct Event Recall

\begin{tabular}{llllll}
\hline & \multicolumn{4}{c}{ Retrieval Cue } \\
\cline { 2 - 5 } \cline { 5 - 6 } Elaboration & \multicolumn{2}{c}{ Boundary } & & \multicolumn{2}{c}{ Map } \\
\cline { 2 - 5 } \cline { 5 - 6 } Nonmodified & Test 1 & Test 2 & & Test 1 & Test 2 \\
$M$ & 7.78 & 4.83 & & 9.25 & 7.12 \\
$S D$ & 3.15 & 2.41 & 1.77 & 2.33 \\
Modified & & & & \\
$M$ & 8.77 & 5.78 & 9.94 & 7.75 \\
$S D$ & 3.17 & 3.42 & 2.46 & 2.69 \\
\hline
\end{tabular}

the expected direction, with map $>$ boundary, elaborated $>$ nonelaborated, and immediate $>$ delay.

A 2 (map) $\times 2$ (occasion) analysis of the number of modifiers recalled produced a significant outcome only for the occasion main effect $\left[F(1,32)=35.84, M S_{e}=\right.$ 3.33]. Averages of 7.00 and 4.38 modifiers were recalled on the immediate and delay test, respectively. Of the modifiers actually recalled, $10 \%$ were size and shape descriptors, with material and color accounting for $42 \%$ and $48 \%$, respectively. Hence, the recall emphasis was on adjectives modifying the visual texture of the features.

We next analyzed the conditional relationship between recall of modifiers and their associated events. Our first set of conditional probabilities was of the form $P$ (elaborated event $\mid$ modifier), where the values were calculated separately for immediate and delay tests. A 2 (map) $\times 2$ (occasion) analysis on this data was significant only for the occasion main effect $\left[F(1,31)=18.89, M S_{\mathrm{e}}=.04\right]$. The mean probabilities were .76 (immediate), and .54 (delay). The second set of conditional probabilities was of the form $P$ (nonelaborated event $\mid$ modifier), and here the same analysis produced significant main effects for map $[F(1,31)=$ $\left.4.92, M S_{\mathrm{e}}=.11\right]$ and occasion $\left[F(1,31)=11.79, M S_{\mathrm{e}}=\right.$ $.02]$, but their interaction was not significant. The mean probabilities for the map condition were .53 and .41 , and for the boundary group .34 and .23 , for the immediate and delay tests, respectively. As expected, event recall probability was significantly higher when the modifier was recalled as opposed to when it was not for both the immediate $\left[F(1,28)=13.24, M S_{\mathrm{e}}=.05\right]$ and the delay tests $\left[F(1,27)=13.83, M S_{\mathrm{e}}=.04\right]$.

The results of the imagery and location ratings were disappointing. Overall, imagery ratings $(M=2.84)$ were higher than location ratings $[M=2.63 ; F(1,32)=11.74$, $\left.M S_{\mathrm{e}}=.12\right]$ and ratings were generally lower on the delayed test $\left[F(1,32)=95.31, M S_{\mathrm{e}}=.21\right]$. The ratings failed to correlate significantly with any of the remaining variables in the study.

The subjects in the boundary condition recalled a large proportion of features within the framework of the map boundary. The average numbers of features correctly identified and located were 12.44 ( $83 \%$ of total) for the immediate test and $9.44(63 \%)$ for the delay measure.

\section{DISCUSSION}

As the conjoint retention argument predicts, event recall was higher for the map condition. One possible explanation for this result is that the perceptually available features serve a simple cuing function during free recall. In other words, superior recall by subjects who saw the map is due simply to the availability of feature names as cues for remembering events. We consider this explanation unlikely, however, since the subjects in the boundary condition were able to accurately locate $83 \%$ of the features on the immediate test. This result indicates that the boundary subjects had a large proportion of the feature array accurately represented in memory. The location of features dropped to $63 \%$ on the delay measure, but this decrease had no differential effect on recall between groups-there was no map $x$ occasion interaction. Hence, we are satisfied that the higher event recall of the map group was due to referential access, particularly with the high amount of detail contained in the feature icons.

The recall of modifying adjectives was about the same for the two map groups. Recall of a modifier was more strongly linked to recall of the event directly associated with the modifier than with the event where the feature name was not modified. However, events that occurred with a nonmodified feature were significantly better remembered when the modifier itself was recalled and the perceptual form of the feature was available. This outcome is in line with our earlier argument concerning the additivity of visual detail. When the feature is available with high detail as in the icons used here, and the detail is increased by the addition of visual texture (color and material), the probability increases that activation from the feature label will fan out over greater event territory.

\section{REFERENCES}

Abel, R. R., \&ulhavy, R. W. (1986). Maps, mode of text presentation, and children's prose learning. American Educational Research Journal, 23, 263-274.

Dean, R. S., \& Kulhavy, R. W. (1981). Influence of spatial organization on prose learning. Journal of Educational Psychology, 73, $57-64$.

Kulhavy, R. W., Lee, J. B., \& Caterino, L. C. (1985). Conjoint retention of maps and related discourse. Contemporary Educational Psychology, 10, 28-37.

LARKIN, J. H., \& SimON, H. A. (1987). Why a diagram is (sometimes) worth ten thousand words. Cognitive Science, 11, 65-99.

PAIvio, A. (1986). Mental representations: A dual coding approach. New York: Oxford University Press.

Shank, G. (1990). Chanson de real world. Tallahassee, FL: National Consortium for Instruction and Cognition.

(Manuscript received October 13, 1990.) 